\title{
Efeitos cardiorrespiratórios da metadona, pelas vias intramuscular e intravenosa, em cadelas submetidas à ovariossalpingo-histerectomia
}

\author{
[Cardiorespiratory effects of methadone, by intramuscular or intravenous injection, in female \\ dogs submitted to ovariohysterectomy] \\ D.A. Pereira ${ }^{1}$, J.A. Marques ${ }^{2}$, P.A. Borges ${ }^{1}$, P.A.C.S. Batista ${ }^{1}$, C.A. Oliveira ${ }^{3}$, \\ N. Nunes ${ }^{2}$, P.C.F. Lopes ${ }^{1}$ \\ ${ }^{1}$ Aluna de pós-graduação - Unesp - Jaboticabal, SP \\ ${ }^{2}$ Faculdade de Ciências Agrárias e Veterinárias - Unesp - Jaboticabal, SP \\ ${ }^{3}$ Faculdade de Ciências Veterinárias e Zootecnia - Universidade de São Paulo, SP
}

\begin{abstract}
RESUMO
A metadona é um opioide pouco utilizado na medicina veterinária, e há, ainda, questionamentos quanto ao seu uso. Desse modo, comparou-se o emprego da metadona, administrada pelas vias IM e IV, e avaliaram-se seus efeitos sobre as características cardiorrespiratórias e os tempos de extubação e recuperação em cadelas submetidas à ovariossalpingo-histerectomia. Utilizaram-se 16 cadelas, prémedicadas com levomepromazina, $0.6 \mathrm{mg} / \mathrm{kg}$, IM. Após 20 minutos, a indução anestésica foi feita com propofol, $5 \mathrm{mg} / \mathrm{kg}$, IV, e a manutenção com isoflurano. Transcorridos 10 minutos, os animais receberam metadona, $0.3 \mathrm{mg} / \mathrm{kg}$, pelas vias IM - grupo GIM - e IV - grupo GIV. As mensurações das frequências cardíaca (FC) e respiratória (FR), pressão arterial média (PAM), temperatura esofágica (TE), pressão parcial de dióxido de carbono $\left(\mathrm{EtCO}_{2}\right)$ e saturação de oxi-hemoglobina $\left(\mathrm{SpO}_{2}\right)$ foram registradas antes da administração do opioide (T0), após 20 minutos (T1) e a cada 10 minutos da administração até 60

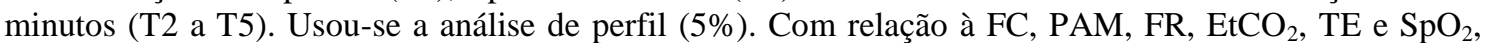
não foram observadas diferenças significativas entre grupos e momentos. Observaram-se tempos de extubação e recuperação maiores no GIV. É possível concluir que a administração IV e a IM da metadona não produzem alterações nas características cardiorrespiratórias em cadelas.
\end{abstract}

Palavras-chave: cadela, anestesia, analgesia, metadona, ovário-histerectomia

\begin{abstract}
Methadone is a little used opioid in veterinary practice, and there are still questions about its use. The objective of this study was to evaluate the effects of intramuscular (IM) or intravenous (IV) administration of methadone on cardiopulmonary parameters and times of extubation and recovery in female dogs submitted to ovariohysterectomy. Sixteen adult female dogs were used and premedicated with levomepromazine $(0.6 \mathrm{mg} / \mathrm{kg}$, IM). After 20 minutes, propofol $(5 \mathrm{mg} / \mathrm{kg}, I V)$ was used for induction and anesthesia was maintained with isoflurane. After 10 minutes, methadone at $0.3 \mathrm{mg} / \mathrm{kg}$ was administered intravenously in IVG and intramuscularly in IMG. The measurement of heart (HR) and respiratory rates $(R R)$, median arterial pressure $(M A P)$, esophagic temperature $(E T)$ and concentration of end-tidal carbon dioxide $\left(\mathrm{PE}^{\prime} \mathrm{CO}_{2}\right)$ was performed immediately before the administration of the opioid (TO), after 20 minutes (T1) and then at 10-minute intervals (T2, T3, T4 and T5). The statistical analysis used was profile (5\%). HR, APM, RR, $\mathrm{PE}^{\prime} \mathrm{CO}_{2}, \mathrm{BT}$ and $\mathrm{SpO}_{2}$ did not differ significantly among times or between groups at any time. Times of extubation and recovery were higher in IVG. It is possible to conclude that IV and IM administration of methadone did not produce changes in the cardiorespiratory parameters of that specie.
\end{abstract}

Keywords: bitch, anesthesia, analgesia, methadone, ovariohisterectomy

Recebido em 2 de dezembro de 2011

Aceito em 7 de fevereiro de 2013

E-mail: daniveterinaria78@yahoo.com.br 


\section{INTRODUÇÃO}

A analgesia é um componente importante da anestesia geral, e, atualmente, os opioides constituem a classe de medicamentos mais comumente utilizada na medicina veterinária (Mathews et al., 1996). A metadona é uma substância que apresenta efeitos farmacológicos similares aos da morfina, pois atua em receptores do tipo $\mu$, sendo este o seu provável mecanismo analgésico (Gutstein e Akil, 2001). Acredita-se que possa atuar como antagonista de receptores NMDA na medula espinhal, característica que pode incrementar a analgesia proporcionada pelo fármaco e diminuir a ocorrência de tolerância com seu uso contínuo (Wagner, 2002).

Stanley et al. (1980) relataram que a administração de doses cumulativas de metadona, 0,3 a $5,3 \mathrm{mg} / \mathrm{kg}$, IV, resultaram em alterações cardiovasculares pouco significativas em cães. Robinson et al. (1988) descreveram que, em geral, os opioides proporcionam estabilidade cardiovascular, no entanto alterações nos níveis de catecolaminas e histamina podem promover alterações hemodinâmicas induzidas por estes fármacos (Hellebrekers et al., 1987). Ambrisko et al. (2005) relataram que os efeitos hemodinâmicos dos opioides podem ser influenciados pela coadministração de outros fármacos, resultando em maior depressão cardiorrespiratória.

O presente estudo teve como objetivo avaliar os efeitos obtidos pela administração intramuscular e intravenosa da metadona, sobre as características cardiorrespiratórias de cadelas submetidas à ovariossalpingo-histerectomia.

\section{MATERIAL E MÉTODOS}

Este estudo foi previamente aprovado pela Comissão de Ética e Experimentação Animal da Faculdade de Ciências Agrárias e Veterinárias da Unesp Jaboticabal (protocolo $n^{\circ}$ 020919/09).

Foram utilizadas 16 cadelas, sem raça definida e média de peso de $10 \pm 3.4 \mathrm{~kg}$, com idades entre oito e 10 anos, hígidas, as quais foram submetidas à ovariossalpingo-histerectomia. A escolha dos animais foi baseada em exames clínicos e laboratoriais, como hemograma, ureia, creatinina, fosfatase alcalina e ALT, juntamente com o eletrocardiograma. $\mathrm{O}$ procedimento cirúrgico foi autorizado pelo proprietário por meio de assinatura de termo de responsabilidade de guarda.

Os animais foram distribuídos, aleatoriamente, em dois grupos, GIM e GIV, com oito animais cada. Todos os cães foram pré-medicados com levomepromazina (Neozine $\AA$, Rhodia Farma Ltda., Jurubatuba, São Paulo, Brasil), na dose de $0.6 \mathrm{mg} / \mathrm{kg}$ (IM). Após 15 minutos, foram realizadas tricotomias nas regiões da veia cefálica. Subsequentemente, os cateteres (Cateter BD Angiocath $\AA$, Becton Dickinson Indústria Cirúrgica Ltda., Juiz Fora, MG) foram introduzidos nas veias cefálica direita e esquerda, para infusão de ringer lactato (Solução Ringer Lactato, JP Indústria Farmacêutica, Ribeirão Preto, SP), $10 \mathrm{~mL} / \mathrm{kg} / \mathrm{h}$, durante o procedimento.

A indução anestésica foi realizada com propofol (Propovan ${ }^{\circledR} \quad 10 \mathrm{mg} / \mathrm{mL}$, Cristália Produtos Químicos e Farmacêuticos Ltda., São Paulo, SP), $5 \mathrm{mg} / \mathrm{kg}$, IV, na dose necessária para perda do reflexo laringotraqueal. Após a intubação endotraqueal, a manutenção anestésica foi feita com isoflurano (Forane ${ }^{\circledR}$, Cristália, São Paulo, $\mathrm{SP}$ ), diluído em oxigênio, com fluxo de 50 a $100 \mathrm{~mL} / \mathrm{kg} / \mathrm{min}$, utilizando-se um circuito anestésico com reinalação parcial de gases, e o vaporizador (OHMEDA- mod. ISOTEC 5- Datex Ohmeda- Miami, EUA) foi ajustado para manter um plano de anestesia cirúrgica baseada no esquema de Guedel - estágio III, $3^{\circ}$ plano. Os cães foram posicionados em decúbito lateral direito sobre colchão térmico ativo (Colchão Térmico Brasmed, Recanto do Lago, Paulínia, SP). Em seguida, foi cateterizada a artéria femoral do membro pélvico esquerdo, por punção transcutânea, acoplando-se o cateter ao transdutor do monitor multiparamétrico (DIXTAL - mod. Dx2010, módulo PA Invasiva, Manaus, AM, Brasil) para mensuração da pressão arterial.

Após a estabilização da anestesia geral, a metadona (Mytedon ${ }^{\circledR}$ - cloridrato de metadona, Cristália Prod. Químicos e Farmacêuticos Ltda., São Paulo, SP) foi administrada em todos os animais dos GIM e GIV, na dose de $0.3 \mathrm{mg} / \mathrm{kg}$, diluída em solução de cloreto de sódio $0.9 \%$ (Cloreto Sódio 0.9\%, Fresenius Kabi Brasil Ltda., Aquiraz, CE), perfazendo um volume total de $0.2 \mathrm{~mL} / \mathrm{kg}$. 
As medidas eletrocardiográficas foram obtidas por meio de eletrocardiógrafo computadorizado (TEB- mod. ECGPC software versão 1.10, São Paulo, SP, Brasil) em derivação DII, a FC foi obtida calculando-se o intervalo de tempo entre dois intervalos R-R, em milissegundos. Foram registradas a duração (Pms) e a amplitude da onda $\mathrm{P}(\mathrm{PmV})$, em milissegundos e milivolts, respectivamente, a duração do intervalo entre as ondas P e R (PR) e do complexo QRS (QRS), a amplitude da onda $\mathrm{R}(\mathrm{RmV})$ e a duração do intervalo QT e RR, enquanto a frequência respiratória (FR), a pressão parcial de dióxido de carbono no final da expiração $\left(\mathrm{EtCO}_{2}\right)$ e a saturação de oxi-hemoglobina foram obtidas por leitura direta em monitor multiparamétrico, cujo sensor foi conectado entre a sonda orotraqueal e o equipamento de anestesia.

A temperatura esofágica foi mensurada em graus Celsius, por meio de termômetro esofágico digital (Termômetro esofágico digital Dixtal, mod. Dx2010, Manaus, AM, Brasil), posicionado no esôfago, à altura do coração.

As primeiras mensurações foram realizadas imediatamente antes da administração da metadona (T0), com 20 minutos da administração (T1) e a cada 10 minutos da administração até 60 minutos (T2 a T5). Ao término do procedimento cirúrgico, os animais foram conduzidos à sala de recuperação, onde foram observados o tempo médio para extubação e o período e qualidade de recuperação anestésica. Ao término do protocolo experimental, os animais receberam antibioticoterapia com benzilpenicilina benzatina (Multibiótico Veterinário, Indústria Farmacêutica Vitalfarma Ltda., MG), na dose de 40000UI/kg, IM, em intervalos de 24 horas, durante dois dias, flunixim meglumine (Banamine, ScheringPlough Veterinária, Rio de Janeiro, RJ, Brasil) na dose de $1,1 \mathrm{mg} / \mathrm{kg}$, por via oral, uma vez ao dia, durante três dias, e tramadol (Tramadol, Cristália Produtos Químicos e Farmacêuticos Ltda., São Paulo, SP, Brasil), na dose de $2 \mathrm{mg} / \mathrm{kg}$, IM, três vezes ao dia, durante dois dias.

\section{RESULTADOS E DISCUSSÃO}

Neste trabalho, foi possível observar que, em dois animais do GIV (25\%), imediatamente após a administração do opioide, houve a ocorrência de bloqueios atrioventriculares, contrações ventriculares prematuras e bradicardia. Conforme Ebert et al. (1995), este resultado ocorreu devido aos efeitos cronotrópicos negativos da metadona, os quais podem ser atribuídos ao aumento do tônus vagal. Segundo Stanley et al. (1980), em cães não houve alteração cardiovascular durante ou após a administração da metadona nas doses de 0,3 ou $0,5 \mathrm{mg} / \mathrm{kg}$, semelhantemente, neste estudo, mudanças não foram verificadas na FC (Fig. 1).

Quanto aos traçados eletrocardiográficos, para as variáveis Pms, PR, QRS, RmV, QT e RR, não foram observadas alterações importantes entre os grupos (Fig. 3), e verificou-se que, nas condições deste experimento, a metadona, juntamente com o isoflurano, não influenciou de maneira expressiva a condutibilidade cardíaca destes animais durante os momentos analisados. Já para a variável $\mathrm{PmV}$, foi registrada diferença entre grupos em T4, pois, de acordo com relatos de Raatikainen et al. (1998), os opioides e agentes inalatórios podem produzir alterações no ECG em cães e gatos, conforme as doses utilizadas.

A depressão respiratória pode ser causada pela administração de opioides quando estes são coadministrados com potentes agentes depressores respiratórios, como os anestésicos (Wagner, 2002). Entretanto, neste estudo, nenhuma alteração foi observada para as variáveis $\mathrm{FR}$ e $\mathrm{EtCO}_{2}$ entre grupos e tempos durante todo o procedimento (Fig. 6 e 7). Já para a variável $\mathrm{SpO}_{2}$, houve diferença entre grupos em T2 (Fig. 4), mas, de acordo com a estabilidade observada para todas estas variáveis, neste estudo, independentemente da via de administração, a metadona não causou depressão respiratória, semelhantemente aos achados de Bley et al. (2004), os quais não observaram depressão do sistema respiratório em gatos que receberam o mesmo fármaco pela via intramuscular. 


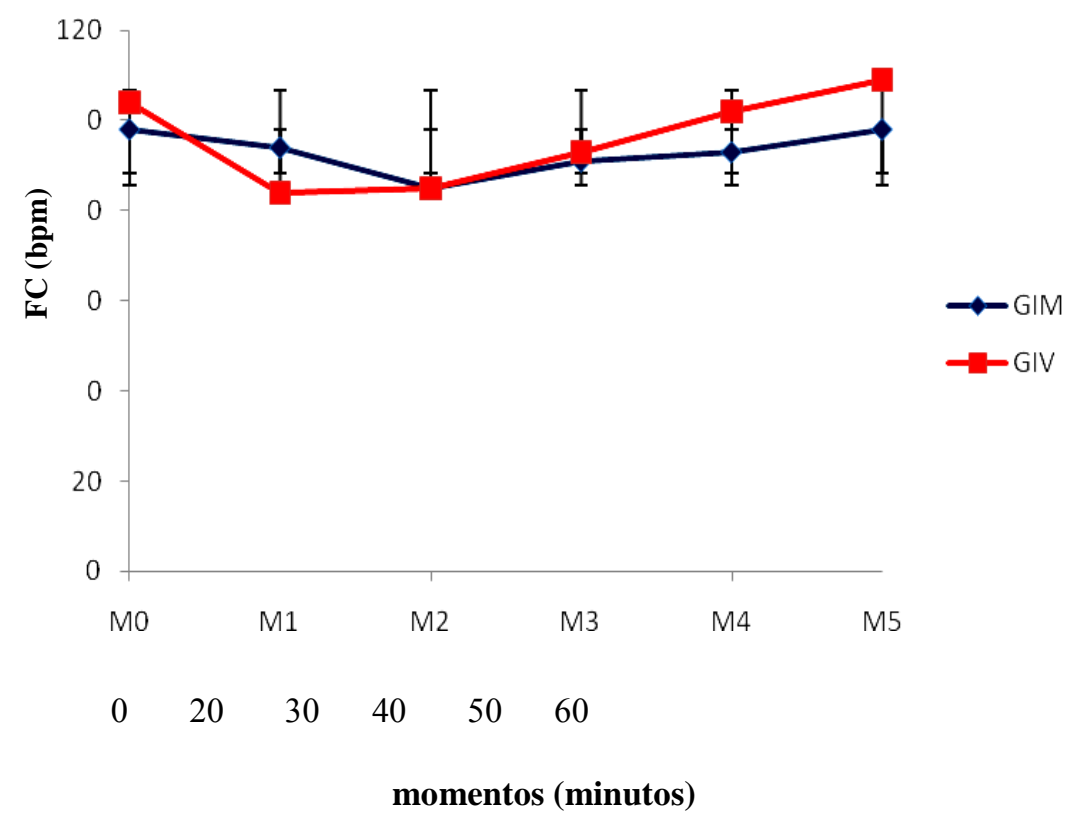

Figura 1. Representação dos valores médios de frequência cardíaca (FC) em cadelas (n=16), tratadas com metadona administrada por via intramuscular (GIM) e intravenosa (GIV), em diferentes momentos, durante ovariossalpingo-histerectomia.

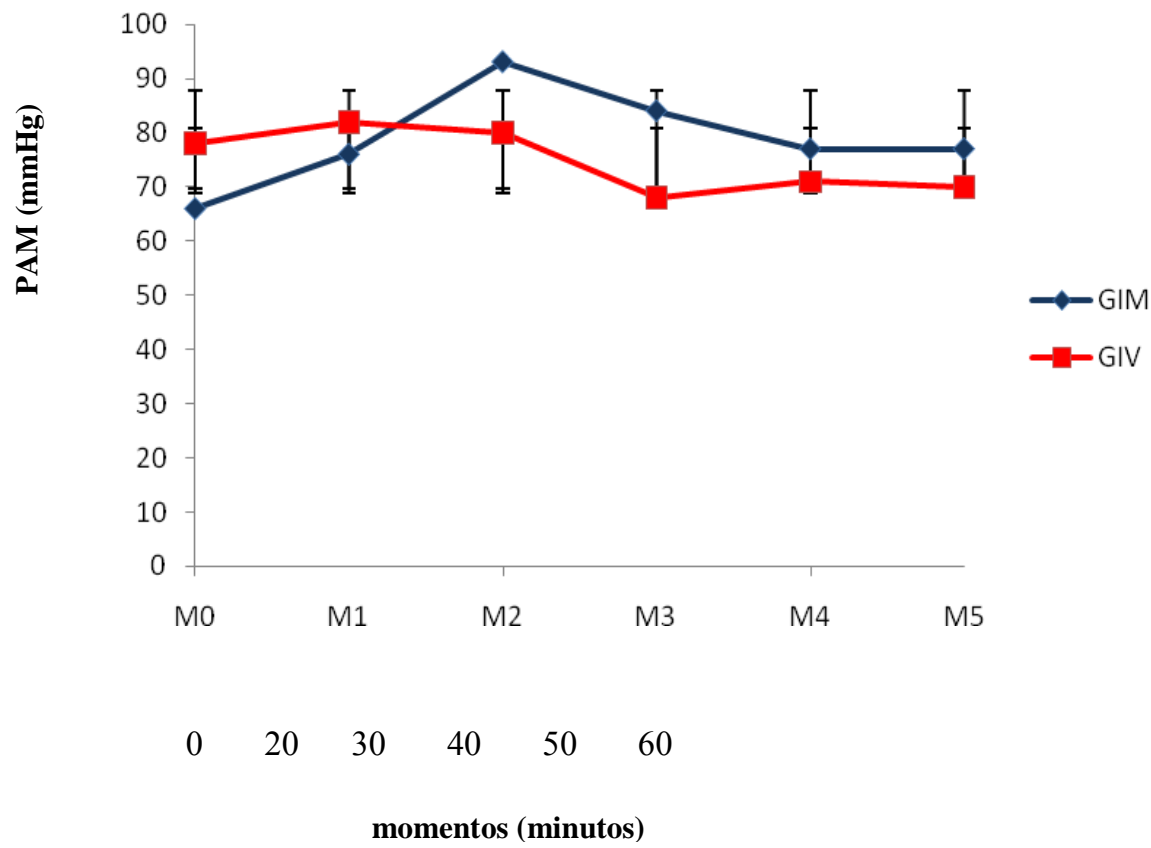

Figura 2. Representação dos valores médios de pressão arterial média (PAM) em cadelas ( $\mathrm{n}=16)$, tratadas com metadona administrada por via intramuscular (GIM) e intravenosa (GIV), em diferentes momentos, durante ovariossalpingo-histerectomia. 


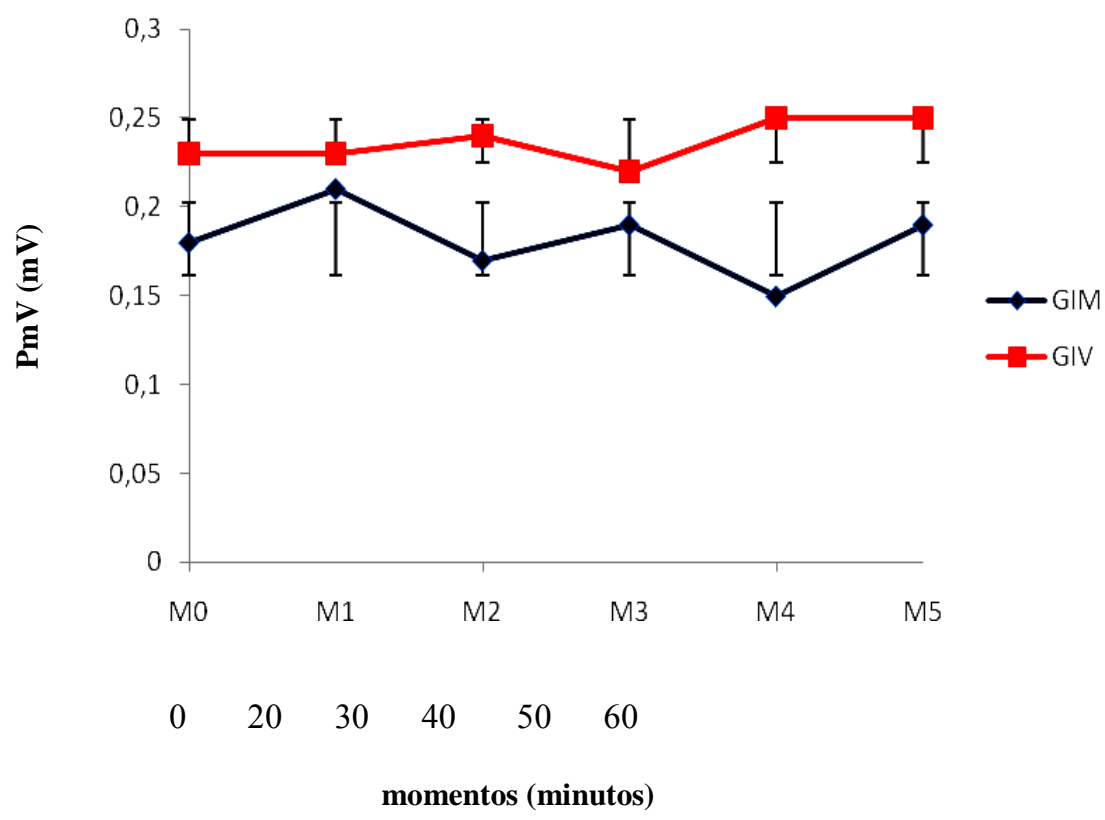

Figura3. Representação dos valores médios da amplitude da onda $\mathrm{P}(\mathrm{PmV})$ em cadelas $(\mathrm{n}=16)$, tratadas com metadona administrada por via intramuscular (GIM) e intravenosa (GIV), em diferentes momentos, durante ovariossalpingo-histerectomia.

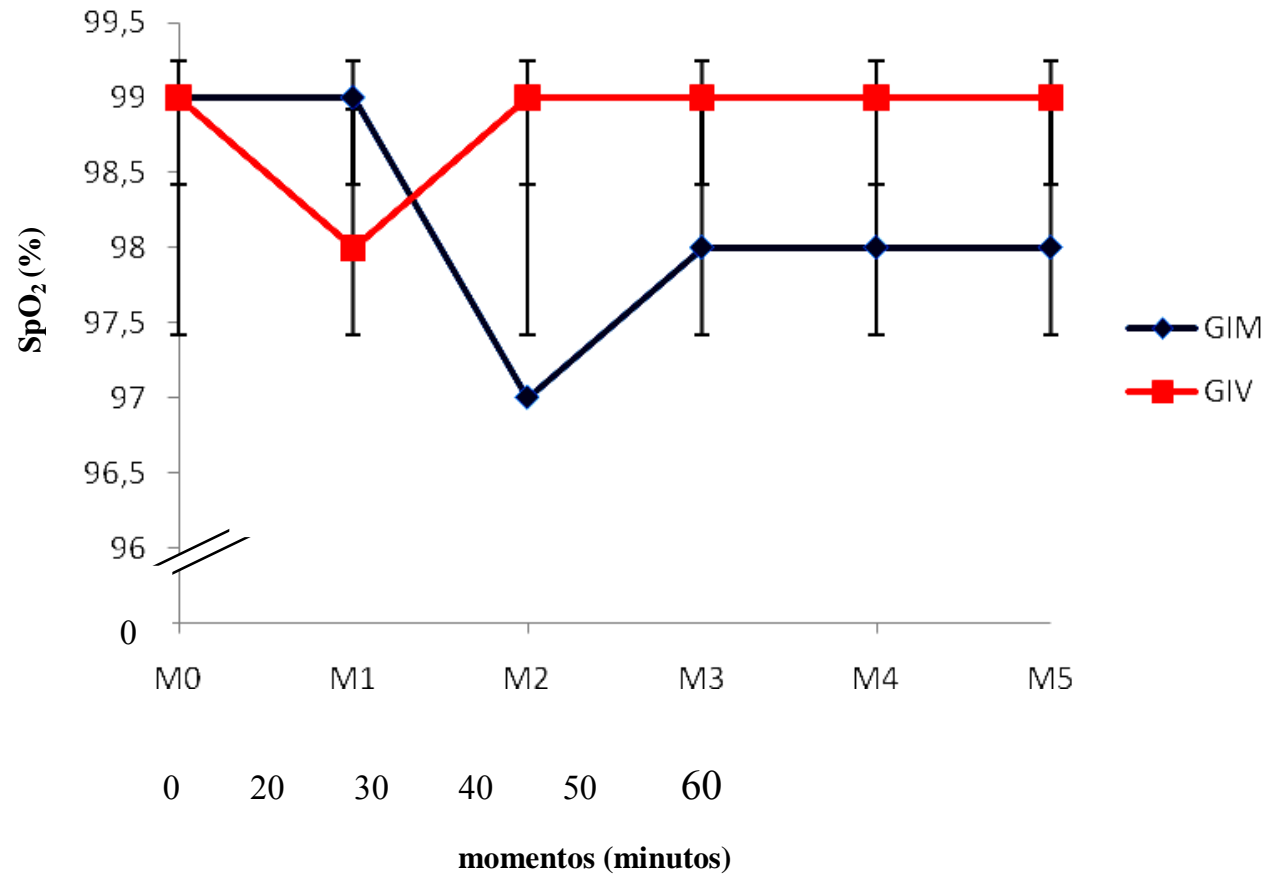

Figura 4. Representação dos valores médios da saturação de oxi-hemoglobina $\left(\mathrm{SpO}_{2}\right)$ em cadelas $(\mathrm{n}=16)$, tratadas com metadona administrada por via intramuscular (GIM) e intravenosa (GIV), em diferentes momentos, durante ovariossalpingo-histerectomia. 


\section{Pereira et al.}

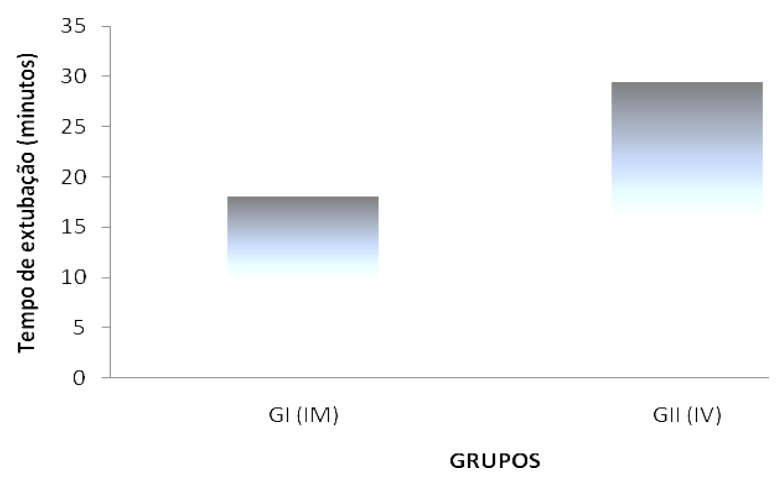

Figura 5. Representação dos valores médios do tempo de extubação (Te) em cadelas (n=16), tratadas com metadona administrada por via intramuscular (GIM) e intravenosa (GIV), após ovariossalpingohisterectomia.

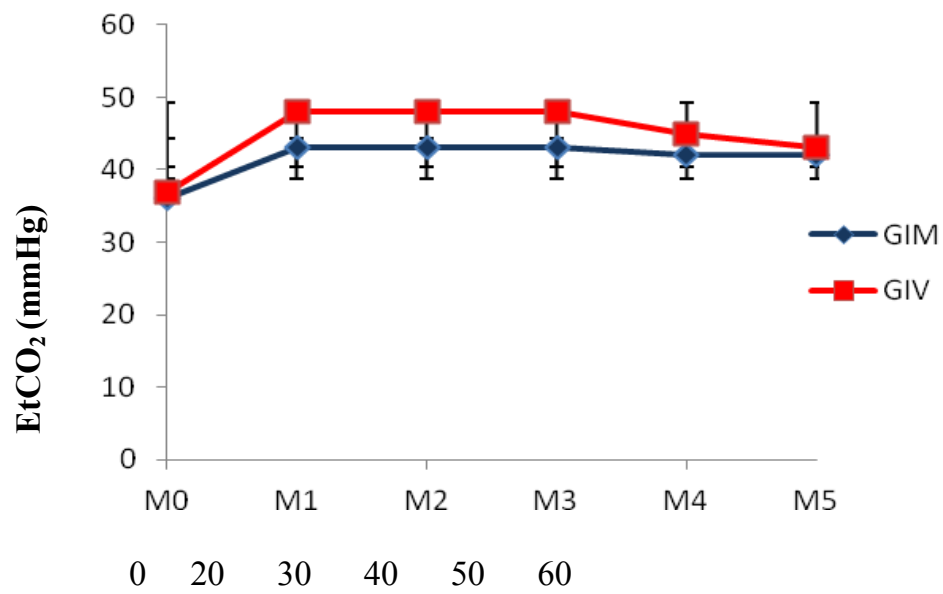

Figura 6. Representação dos valores médios da pressão parcial de dióxido de carbono no final da expiração $\left(\mathrm{EtCO}_{2}\right)$ em cadelas $(\mathrm{n}=16)$, tratadas com metadona administrada por via intramuscular $(\mathrm{GIM})$ e intravenosa (GIV), em diferentes momentos, durante ovariossalpingo-histerectomia.

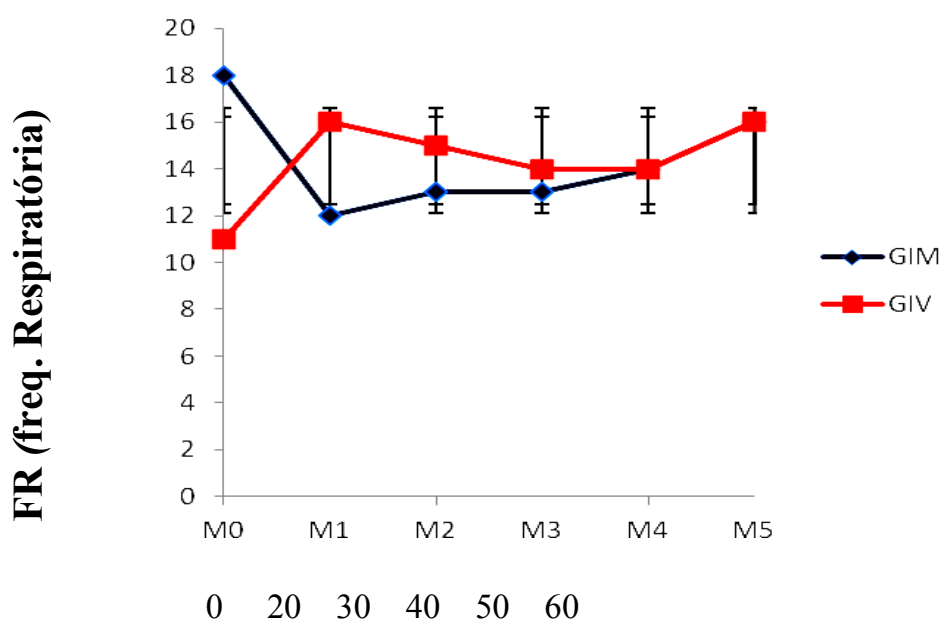

Figura 7. Representação dos valores médios da frequência respiratória (FR) em cadelas $(n=16)$, tratadas com metadona administrada por via intramuscular (GIM) e intravenosa (GIV), em diferentes momentos, durante ovariossalpingo-histerectomia. 
Nunes et al. (1995) citam que a anestesia é comumente associada a um decréscimo progressivo na temperatura corporal e se deve a muitos fatores, incluindo vasodilatação periférica, radiação sobre superfície corporal, decréscimo no metabolismo basal, condução e evaporação. Adicionalmente, o sistema termorregulatório hipotalâmico também é afetado pela administração de opioides (Lamont et al., 2007). No presente estudo, entretanto, para a temperatura esofágica, não houve diferenças significativas registradas. Esse evento pode ser atribuído ao uso do colchão térmico durante todo o procedimento.

Ao longo deste estudo, não houve qualquer alteração nas pressões arteriais (Fig. 2), e essa estabilidade das pressões verificadas ao longo dos momentos está relacionada à baixa dose utilizada e aos mínimos efeitos do opioide e do agente anestésico inalatório sobre a pressão sanguínea, sendo que essas substâncias podem promover diminuição da $\mathrm{PA}$, à medida que suas concentrações ou doses são aumentadas (Clarke, 1996).

De acordo com Leibetseder et al. (2006), não foram verificadas diferenças entre os tempos de extubação em cães submetidos às injeções IV e extradural de metadona; resultado semelhante foi relatado por Bley et al. (2004) em gatas tratadas com o mesmo opioide, diferindo dos dados deste estudo, em que foi observado que, para Te, houve diferença entre grupos, demonstrando maior tempo de extubação nos animais do GIV. Foi observada diferença significativa entre grupos, durante o tempo de recuperação, sendo a média do GIV maior que a do GIM (Fig. 5). Estes achados podem ser atribuídos ao efeito sedativo dos opioides como resultado da interação com receptores $\mu$ e $\kappa$, mas outros fatores devem interferir na sedação, tais como a dose e as diferenças na farmacocinética e na variação individual, sendo esses parâmetros influenciados pelas diferentes vias de administração (Muir, 2002).

\section{CONCLUSÕES}

Sugere-se que a administração da metadona, na dose de $0,3 \mathrm{mg} / \mathrm{kg}$, pelas vias IM e IV, promove alterações cardiorrespiratórias discretas e poucos efeitos adversos, não sendo indicado seu uso pela via parenteral, em animais debilitados ou suscetíveis a instabilidades cardiovasculares.

\section{REFERÊNCIAS}

AMBRISKO, T.D.; HIKASA, Y.; SATO, K. Influence of medetomidine on stress-related neurohormonal and metabolic effects caused by butorphanol, fentanil and ketamine administration in dogs. Am. J. Vet. Res., v.66, p.406-412, 2005.

BLEY, C.R.; NEIGER-AESCHBACHER, G.; BUSATO, A. et al. Comparison of perioperative racemic methadone, levo-methadone and dextromoramide in cats using indicators of postoperative pain. Vet. Anaesth. Analg., v.31, p.175182, 2004.

CLARKE, K.W. Cardiopulmonary effects of desflurane in the dog during spontaneous and artificial ventilation. Res. Vet. Sci., v.61, p.82-86, 1996.

EBERT, B.; ANDERSEN, S.; KROGSGAARDLARSEN, P. Ketobemidone, methadone and pethidine are non-competitive N-methyl-Daspartate antagonists in the rat cortex and spinal cord. Neur. Letters, v.187, p.165-168, 1995.

GUTSTEIN, H.B.; AKIL, H. Opioid analgesics. In: HARDMAN, J.G.; LIMBIRD, L.E. Goodman \& Gilman's: the pharmacological basis of therapeutics. 10.ed. New York: McGraw-Hill, 2001. p.569-619.

HELLEBREKERS, L.J.; MOL, J.A.; VAN DEN BROM, W.E. et al. Effect of methadone on plasma arginine vasopressin level and urine production in conscious dogs. Eur. J. Pharmacol., v.136, p.279-286, 1987.

LAMONT, L.A.; MATHEWS, K.A. Opioids, nonsteroidal antiinflamatories and analgesic adjuvantes. In: TRANQUILLI, W.J.; THURMON, J.C.; GRIM. K.A. Vet. Anesth. Analg. 4. Ed. Sydney: Blackwell Publishing, 2007. p.241-271. 
LEIBETSEDER, E.N.; MOSING, M.; JONES, R.S. A comparison of extradural and intravenous methadone on intraoperative isoflurane and postoperative analgesia requirements in dogs. Vet. Anaesth. Analg., v.33, p.128-136, 2006.

MATHEWS, K.A.; PALEY, D.M.; FOSTER, R.A. et al. A comparison of ketorolac with flunixin, butorphanol and oxymorphone in controlling postoperative pain in dogs. Can. Vet. J., v.37, p.557-567, 1996.

MUIR, W.W. Drugs used to treat pain. In: GAYNOR, J.S.; MUIR, W.W. Handbook Veterinary Pain Management. St Louis: Mosby, 2002. p.142-163.

NUNES, N.; POMPERMAYER, L.G.; PIROLO, J. et al. Emprego do metaraminol no bloqueio da hipotensão produzida pelo uso da levomepromazina em cães. Braz. J. Vet. Res. Anim. Sci., v.32, p.120-124, 1995.
RAATIKAINEM, M.J.; TRANKINA, M.F.; MOREY, T.E. et al. Effects of volatile anesthetics on atrial and AV nodal electrophysiological properties in guinea pig isolated perfused heart. Anesthesiol., v.89, p.434442, 1998.

ROBINSON, E.P.; FAGGELLA, A.M.; HENRY, D.P. et al. Comparison of histamine released induced by morphine and oxymorphone administration in dogs. Am. J. Vet. Res., v.49, p.1699-1701, 1988.

STANLEY, T.H.; LIU, W.S.; WEBSTER, L.R. et al. Haemodynamic effects of intravenous methadone anaesthesia in dogs. Can. Anaesth. Soc. J., v.27, p.53-57, 1980.

WAGNER, A.E. Opioids. In: GAYNOR, J.S; MUIR, W.W. Handbook Of Veterinary Pain Management. 2. Ed. Saint Louis: Mosby Elsever, 2002. p.163-182. 